Mathematical Modelling and Analysis

Volume 22 Number 4, July 2017, 528-547

https://doi.org/10.3846/13926292.2017.1329757

(C) Vilnius Gediminas Technical University, 2017
Publisher: Taylor\&Francis and VGTU

http://www.tandfonline.com/TMMA

ISSN: $1392-6292$

eISSN: $1648-3510$

\title{
Solvability of a Class of $N$-th Order Linear Focal Problems
}

\author{
Pedro Almenar ${ }^{a}$ and Lucas Jódar ${ }^{b}$ \\ ${ }^{a}$ Vodafone Spain \\ Avda. América 115, 28042 Madrid, Spain \\ ${ }^{b}$ Instituto Universitario de Matemática Multidisciplinar, Universitat \\ Politècnica de València \\ Camino de Vera s/n, 46022 Valencia, Spain \\ E-mail(corresp.): pedro.almenar1@vodafone.com \\ E-mail: 1jodar@imm.upv.es
}

Received January 8, 2017; revised May 7, 2017; published online July 15, 2017

\begin{abstract}
This paper presents a recursive method which yields necessary and sufficient conditions for the existence of solutions of a class of $n$-th order linear focal boundary value problems in the interior of a given interval, in the form of integral inequalities. Some results on the sign of the derivatives of the Green functions of $n$-th order linear focal boundary value problems will also be provided.
\end{abstract}

Keywords: disfocality, n-th order linear boundary value problem, Green function, cone theory, Lyapunov inequality.

AMS Subject Classification: 34B05; 34B09; 34B27; 34C10; 47B65; 47N20.

\section{Introduction}

Let $I$ be a compact interval in $\mathbb{R}$, let $k, n \in \mathbb{N}$ be such that $1 \leq k<n$ and let us consider the $n$-th order boundary value problem

$$
\begin{aligned}
& \left.y^{(n)}+p_{n-1}(x) y^{(n-1)}+\ldots+p_{0}(x) y=0, \quad x \in\right] a^{\prime}, b^{\prime}[ \\
& y^{(i)}\left(a^{\prime}\right)=0, \quad i=0,1,2, \ldots, k-1, \\
& y^{\left(\beta_{i}\right)}\left(b^{\prime}\right)=0, i=1,2, \ldots, n-k, 0 \leq \beta_{1}<\beta_{2}<\ldots<\beta_{n-k}=n-1,
\end{aligned}
$$

where $\left[a^{\prime}, b^{\prime}\right] \subset I$ and $p_{j}(x), 0 \leq j \leq n-1$, are functions continuous on $I$. The purpose of this paper is to describe a method that allows asserting the existence (or not) of solutions of (1.1)-(1.3) in extremes $a^{\prime}, b^{\prime}$ interior to a given interval $[a, b] \subset I$, under certain conditions to be determined. Examples of these types of problems appear in the study of the deflections of beams, both straight ones with non-homogeneous cross-sections in free vibration, which are subject to the fourth-order linear Euler-Bernoulli equation, and curved ones 
with different shapes. An account of these and other applications can be found in $[10$, Chapter IV].

To this end, let us note that we can rewrite the problem (1.1)-(1.3) as

$$
\begin{aligned}
& \left.L y=\sum_{i=0}^{\mu} c_{i}(x) y^{(i)}(x), \quad x \in\right] a^{\prime}, b^{\prime}[, \\
& y^{(i)}\left(a^{\prime}\right)=0, \quad i=0,1,2, \ldots, k-1, \\
& y^{\left(\beta_{i}\right)}\left(b^{\prime}\right)=0, i=1,2, \ldots, n-k, 0 \leq \beta_{1}<\beta_{2}<\ldots<\beta_{n-k}=n-1,
\end{aligned}
$$

where $L: C^{n}(I) \rightarrow C(I)$ is the right-disfocal differential operator defined by

$$
L y=y^{(n)}(x)+a_{n-1}(x) y^{(n-1)}(x)+\cdots+a_{0}(x) y(x), \quad x \in I,
$$

$\mu \leq n-1, a_{j}(x) \in C(I), 0 \leq j \leq n-1$ and $c_{i}(x) \in C(I), 0 \leq i \leq \mu$.

We will show that under certain conditions on the sign of $a_{j}(x), c_{i}(x)$ and the values of $\mu$ and $\beta_{1}$, the operator $M: C^{\mu}[a, b] \rightarrow C^{n}[a, b]$ defined by

$$
M f=\int_{a}^{b} G(x, t) \sum_{i=0}^{\mu} c_{i}(t) f^{(i)}(t) d t,
$$

where $[a, b] \subset I$ and $G(x, t)$ is the Green function of the problem

$$
\begin{aligned}
& L y=0, \quad x \in] a, b[ \\
& y^{(i)}(a)=0, \quad i=0,1,2, \ldots, k-1, \\
& y^{\left(\beta_{i}\right)}(b)=0, i=1,2, \ldots, n-k, 0 \leq \beta_{1}<\beta_{2}<\ldots<\beta_{n-k}=n-1,
\end{aligned}
$$

(assuming that such a problem has only the trivial solution, assumption which we will keep throughout the paper), maps a cone of functions into itself, in a manner that its recursive application over functions of the cone yields necessary and sufficient conditions for the existence of solutions of the problem (1.4)(1.7) for $a^{\prime}$ and $b^{\prime}$ interior to $a, b$, in the form of integral inequalities. The iterative comparison provides lower and upper bounds for the extremes $a^{\prime}$ and $b^{\prime}$ for which (1.4)-(1.6) has a non-trivial solution, bounds which converge to the values of these extremes $a^{\prime}$ and $b^{\prime}$ as the recursivity index grows.

The procedure to prove it will make use of cone theory and in particular $[2$, Theorem 2], as well as certain properties of the sign of the derivatives of the Green function of the problem (1.9). In this way, this paper can be considered as an extension of [2] and [3] to boundary value problems with right focal conditions (these papers requested $\beta_{n-k}<n-1$ in (1.6) as a fundamental assumption).

Let us recall that the application of the cone theory to boundary value problems dates from the works of Krein and Rutman [14] and Krasnosel'skii [13], which were followed by many authors. References [5], [7], [9], [11], [12], [16], [17] and [18] are a good account of this.

In terms of nomenclature, we will say that $a^{\prime}, b^{\prime}$ are interior to $a, b$ if $a \leq a^{\prime}<b^{\prime} \leq b$ and $a<a^{\prime}$ or $b^{\prime}<b$. We will use the notation $M$ to name the operator defined in (1.8), $M^{i} f(M f)$ to name the function resulting of 
the application of $M$ to $f(x) \in C^{\mu}[a, b]$ recursively $i$ (one) times and $M f(x)$ to name the value of the function $M f$ at the point $x$. We will use $G_{a b}(x, t)$ to name the Green function of (1.9) when we want to stress the dependence of such a function with regards to the extremes $a, b$. And we will denote by $P C[a, b]$ the set of piecewise continuous functions on $[a, b]$.

In order to make this paper self-contained, let us recall that, given a Banach space $B$, a cone $P \subset B$ is a non-empty closed set defined by the conditions:

1. If $u, v \in P$ then $c u+d v \in P$ for any real numbers $c, d \geq 0$.

2. If $u \in P$ and $-u \in P$, then $u=0$.

We will denote the interior of the cone $P$ by $P^{0}$, and we will say that the cone $P$ is reproducing if any $y \in B$ can be expressed as $y=u-v$ with $u, v \in P$. The existence of a cone in a Banach space $B$ allows to define a partial ordering relationship in that Banach space by setting $u \leq v$ if and only if $v-u \in P$. Thus, we will say that the operator $M$ is $u_{0}$-positive if there exists a $u_{0} \in P$ such that for any $v \in P \backslash\{0\}$ one can find positive constants $\delta_{1}, \delta_{2}$ such that $\delta_{1} u_{0} \leq M v \leq \delta_{2} u_{0}$ (note that $\delta_{1}$ and $\delta_{2}$ will not be the same for all $v$ ).

The organization of the paper is as follows: Section 2 will provide results on the sign of the Green function of the problem (1.9) and its derivatives. Sections 3 and 4 will describe the method to obtain necessary and sufficient conditions for the problem (1.4)-(1.6) to have a non-trivial solution, Section 3 being dedicated to the case $\beta_{1}<k$ and Section 4 to the case $\beta_{1}=k$. Section 5 will apply the previous results to several examples. Finally Section 6 will elaborate some conclusions.

\section{The sign of the Green function of the problem}

In this section we will obtain some sign properties of the Green functions associated to the problem (1.9). The starting point for this discussion is a theorem of Eloe and Ridenhour [8, Theorem 2.1] which, among other things, provides signs for all derivatives of $G(x, t)$ from 0 to $\min \left(k-1, \beta_{1}\right)$.

Theorem 1. The Green function $G(x, t)$ of the problem (1.9) satisfies

$$
\begin{aligned}
& \left.(-1)^{n-k} \frac{\partial^{i} G(x, t)}{\partial x^{i}}>0, \quad 0 \leq i \leq \min \left(k-1, \beta_{1}\right),(x, t) \in\right] a, b[\times] a, b[; \\
& \left.(-1)^{n-k} \frac{\partial^{k} G(a, t)}{\partial x^{k}}>0, \quad t \in\right] a, b[.
\end{aligned}
$$

Next, following Eloe and Ridenhour [8] let us denote by $H$ the partial derivative of the Green function $G(x, t)$ of the problem (1.9) with regards to the extreme $b$ :

$$
H(x, t)=\frac{\partial G_{a b}(x, t)}{\partial b}, \quad(x, t) \in[a, b] \times[a, b] .
$$

Our first theorem will determine the sign of the function $H(x, t)$ and its partial derivatives with regards to $x$. 
Theorem 2. Let us fix $t \in] a, b[$ and let us suppose that

$$
(-1)^{n(\beta, j)} a_{j}(b) \leq 0, \quad j=0, \ldots, n-1 ; \quad j \neq \beta_{i}, \quad i=1, \ldots, n-k,
$$

where $n(\beta, j)$ denotes the number of components $\beta_{i}$ which exceed $j$. Then

$$
(-1)^{n-k} \frac{\partial^{i} H(x, t)}{\partial x^{i}} \geq 0, \quad 0 \leq i \leq \beta_{1}, \quad x \in[a, b] .
$$

If, in particular, there is a $j \in(0, \ldots, n-1) \backslash\left(\beta_{1}, \beta_{2}, \ldots, \beta_{n-k}\right)$ such that $(-1)^{n(\beta, j)} a_{j}(b)<0$, then

$$
\left.(-1)^{n-k} \frac{\partial^{i} H(x, t)}{\partial x^{i}}>0, \quad 0 \leq i \leq \beta_{1}, \quad x \in\right] a, b[.
$$

Proof. We will follow an approach similar to the one used in [6, Lemma 3.7]. Thus, following the argument of [5, Lemma 3.3] $H(x, t)$ must satisfy the BVP

$$
\begin{aligned}
& L H=0, \quad(x, t) \in[a, b] \times[a, b] \\
& \frac{\partial^{i} H(a, t)}{\partial x^{i}}=0, \quad i=0,1,2, \ldots, k-1, \\
& \frac{\partial^{i} H(b, t)}{\partial x^{i}}=-\frac{\partial^{i+1} G(b, t)}{\partial x^{i+1}}, \quad i=\beta_{j}, j=1, \ldots, n-k .
\end{aligned}
$$

We can tackle the problem (2.6)-(2.8) by fixing $t \in] a, b[$ and writing

$$
H(x, t)=\sum_{l=1}^{n-k} y_{l}(x)
$$

where each $y_{l}(x)$ satisfies

$$
\begin{aligned}
& L y_{l}=0, \quad x \in[a, b] \\
& y_{l}^{(i)}(a)=0, \quad i=0,1,2, \ldots, k-1, \\
& y_{l}^{(i)}(b)=0, i=\beta_{j}, j=1, \ldots, n-k, i \neq \beta_{l} ; y_{l}^{\left(\beta_{l}\right)}(b)=-\frac{\partial^{\beta_{l}+1} G(b, t)}{\partial x^{\beta_{l}+1}} .
\end{aligned}
$$

For those $l$ such that $\beta_{l}+1=\beta_{l+1}$, since the problem (1.9) has only the trivial solution by hypothesis, one must have $y_{l}(x) \equiv 0, x \in[a, b]$. Therefore we will consider only those $l$ for which $\beta_{l}+1 \neq \beta_{l+1}$, which includes at least $\beta_{n-k}$.

Let us focus first on the $y_{l}(x)$ with $l<n-k$. From [8, Lemma 2.4] we know that the Green function of the problem (1.9) satisfies

$$
\left.(-1)^{n\left(\beta, \beta_{l}+1\right)} \frac{\partial^{\beta_{l}+1} G(b, t)}{\partial x^{\beta_{l}+1}}>0, \quad t \in\right] a, b[, \quad l=1, \ldots, n-k-1 .
$$

It is easy to show that $n\left(\beta, \beta_{l}+1\right)=n-k-l$. This gives

$$
(-1)^{n-k-l} y_{l}^{\left(\beta_{l}\right)}(b)=-(-1)^{n-k-l} \frac{\partial^{\beta_{l}+1} G(b, t)}{\partial x^{\beta_{l}+1}}<0, l=1, \ldots, n-k-1 .
$$


We can apply recursively [8, Lemma 2.3] to the problem (2.10) with $l<n-k$ to determine that there are $l-1$ changes of sign between $y_{l}^{\left(\beta_{l}\right)}(x)$ and $y_{l}^{\left(\beta_{1}\right)}(x)$ for a $\delta>0$ and $x \in[b-\delta, b$, which combined with (2.11) and [8, Lemma 2.3] yields

$$
-(-1)^{n-k} y_{l}^{\left(\beta_{1}\right)}(x)=(-1)^{n-k-l+l-1} y_{l}^{\left(\beta_{1}\right)}(x) \leq 0, x \in[a, b], l=1, \ldots, n-k-1 .
$$

And from (2.12) and the boundary conditions of (1.9) at $a$ we finally get to

$$
(-1)^{n-k} y_{l}^{(i)}(x) \geq 0, \quad x \in[a, b], \quad l=1, \ldots, n-k-1, \quad 0 \leq i \leq \beta_{1} .
$$

Let us address now the case of $y_{n-k}(x)$. Given that, from [4, Chapter 3, Section 6], $G(x, t)$ is a solution of $L G=0$ on $[a, t[\cup] t, b]$, from (1.7) one has

$$
\frac{\partial^{n} G(b, t)}{\partial x^{n}}=-a_{n-1}(b) \frac{\partial^{n-1} G(b, t)}{\partial x^{n-1}}-\cdots-a_{0}(b) G(b, t) .
$$

Combining (2.3), (2.14) and [8, Lemma 2.4] one gets to $\frac{\partial^{n} G(b, t)}{\partial x^{n}} \geq 0$. If all $a_{j}(b)=0$ for $j \in(0, \ldots, n-1) \backslash\left(\beta_{1}, \beta_{2}, \ldots, \beta_{n-k}\right)$ then $\frac{\partial^{n} G(b, t)}{\partial x^{n}}=0$. From here, (2.10) and the fact that the boundary value problem (1.9) does not have a non-trivial solution as per hypothesis one has

$$
y_{n-k}(x) \equiv 0 .
$$

On the contrary, if there is a $j \in(0, \ldots, n-1) \backslash\left(\beta_{1}, \beta_{2}, \ldots, \beta_{n-k}\right)$ such that $(-1)^{n(\beta, j)} a_{j}(b)<0$ then $\frac{\partial^{n} G(b, t)}{\partial x^{n}}>0$, which together with $(2.10)$ leads to

$$
y_{n-k}^{(n-1)}(b)=-\frac{\partial^{n} G(b, t)}{\partial x^{n}}<0 .
$$

Applying [8, Lemma 2.3] recursively to (2.10) and using (2.16) it follows

$$
\left.(-1)^{n-k} y_{n-k}^{\left(\beta_{1}\right)}(x)>0, \quad x \in\right] a, b[.
$$

From (2.17) and the boundary conditions of (1.9) at $a$ we finally get to

$$
\left.(-1)^{n-k} y_{n-k}^{(i)}(x)>0, \quad x \in\right] a, b\left[, \quad 0 \leq i \leq \beta_{1} .\right.
$$

The combination of (2.9), (2.13), (2.15) and (2.18) finally gives

$$
\left.(-1)^{n-k} \frac{\partial^{i} H(x, t)}{\partial x^{i}} \geq 0, \quad[x, t] \in[a, b] \times\right] a, b\left[, \quad 0 \leq i \leq \beta_{1},\right.
$$

which is (2.4), whereas (2.5) can be obtained from (2.9), (2.13) and (2.18).

Let us turn our mind to the pure right focal case of (1.9) with $\beta_{1}=k$, namely

$$
\begin{aligned}
& L y=0, \\
& y^{(i)}(a)=0, \quad i=0,1,2, \ldots, k-1, \\
& y^{\left(\beta_{i}\right)}(b)=0,\left(\beta_{1}, \beta_{2}, \ldots, \beta_{n-k}\right)=(k, k+1, \ldots, n-1) .
\end{aligned}
$$


In this concrete case, we can extend the results of Theorem 1 to higher derivatives, as no boundary conditions on the same derivative of $G(x, t)$ apply at $a$ and $b$, which would force a change of sign in the next derivative due to Rolle's theorem. We will do so in the next result.

Theorem 3. Let us assume that

$$
(-1)^{n-k} a_{j}(x) \leq 0, \quad x \in[a, b], \quad j=0, \ldots, k-1
$$

and that

$$
(-1)^{n-k} a_{i}(b)<0
$$

for at least one $i$ such that $0 \leq i \leq k-1$. Let us also assume that

$$
(-1)^{n-j} a_{j}(x) \leq 0, \quad x \in[a, b], \quad j=k, \ldots, n-1 .
$$

Then the Green function of the problem (2.20) satisfies

$$
\begin{aligned}
& \left.\left.\left.(-1)^{n-k} \frac{\partial^{i} G(x, t)}{\partial x^{i}}>0, \quad(x, t) \in\right] a, b\right] \times\right] a, b[, \quad i=0, \ldots, k-1, \\
& (-1)^{n-i} \frac{\partial^{i} G(x, t)}{\partial x^{i}}>0, \quad(x, t) \in[a, b[\times] a, b[, \quad i=k, \ldots, n-1 .
\end{aligned}
$$

Proof. We will only prove (2.25) since (2.24) is immediate from Theorem 1, and we will do it by fixing $t \in] a, b[$ and considering the cases $x \in[t, b[$ and $x \in\left[a, t\left[\right.\right.$ separately. Thus, let us assume that there exists an $x_{n-1} \in[t, b[$ such that

$$
\frac{\partial^{n-1} G\left(x_{n-1}, t\right)}{\partial x^{n-1}} \geq 0
$$

and let us denote by $x_{n-1}^{*}$ the supremum of those $x_{n-1}$. We will prove now that there exists an $\epsilon>0$ such that

$$
\frac{\partial^{n-1} G(x, t)}{\partial x^{n-1}}<0, \quad x \in[b-\epsilon, b[.
$$

From [8, Lemma 2.4] we know that

$$
(-1)^{n-k} \frac{\partial^{i} G(b, t)}{\partial x^{i}}>0, \quad 0 \leq i \leq k-1 .
$$

And from (1.7), (2.20), (2.21), (2.28) and condition (2.22) one gets

$$
\begin{aligned}
\frac{\partial^{n} G(b, t)}{\partial x^{n}} & =-a_{n-1}(b) \frac{\partial^{n-1} G(b, t)}{\partial x^{n-1}}-\cdots-a_{0}(x) G(b, t) \\
& =-a_{k-1}(b) \frac{\partial^{k-1} G(b, t)}{\partial x^{k-1}}-\cdots-a_{0}(x) G(b, t)>0 .
\end{aligned}
$$

By continuity of $\frac{\partial^{n} G(x, t)}{\partial x^{n}}$ at $b$ and Taylor's theorem one has (2.27).

Next, from the definition of $x_{n-1}^{*}$ and (2.27) one must have

$$
\left.\frac{\partial^{n-1} G(x, t)}{\partial x^{n-1}}<0, \quad x \in\right] x_{n-1}^{*}, b[
$$


which, combined with Taylor's theorem and the boundary conditions at $b$, yields

$$
\left.(-1)^{n-i} \frac{\partial^{i} G(x, t)}{\partial x^{i}}>0, \quad x \in\right] x_{n-1}^{*}, b[, \quad k \leq i \leq n-1 .
$$

Applying the mean value theorem and taking into account the definition of $x_{n-1}^{*},(2.26)$ and $(2.27)$ there must exist an $\left.x_{n} \in\right] x_{n-1}^{*}, b[$ such that

$$
\frac{\partial^{n} G\left(x_{n}, t\right)}{\partial x^{n}}<0 .
$$

But (1.7), (2.20), (2.21), (2.23), (2.24) and (2.30) imply that

$$
\left.\frac{\partial^{n} G(x, t)}{\partial x^{n}}=-a_{n-1}(x) \frac{\partial^{n-1} G(x, t)}{\partial x^{n-1}}-\cdots-a_{0}(x) G(x, t) \geq 0, \quad x \in\right] x_{n-1}^{*}, b[,
$$

which contradicts (2.31). Accordingly (2.26) must be wrong and

$$
\frac{\partial^{n-1} G(x, t)}{\partial x^{n-1}}<0, \quad x \in[t, b[,
$$

which again gives

$$
(-1)^{n-i} \frac{\partial^{i} G(x, t)}{\partial x^{i}}>0, \quad x \in[t, b[, \quad k \leq i \leq n-1 .
$$

Let us address the case $x \in[a, t[$. From [4, Chapter 3 , Section 6$]$ we know that the $(n-1)$-th derivative of $G(x, t)$ satisfies

$$
\frac{\partial^{n-1} G(t-, t)}{\partial x^{n-1}}-\frac{\partial^{n-1} G(t+, t)}{\partial x^{n-1}}=-1,
$$

which, together with (2.32)-(2.33) and Taylor's theorem implies that there exists a $\delta>0$ such that

$$
(-1)^{n-i} \frac{\partial^{i} G(x, t)}{\partial x^{i}}>0, \quad x \in[t-\delta, t[, \quad k \leq i \leq n-1 .
$$

Now, let us assume that there exists $j \in(k, \ldots, n-1)$ and $\xi_{j} \in[a, t-\delta[$ with

$$
(-1)^{n-j} \frac{\partial^{j} G\left(\xi_{j}, t\right)}{\partial x^{j}} \leq 0 .
$$

If there are several $j$, let us pick the lowest one. Let us denote by $\xi_{j}^{*}$ the supremum of all $\xi_{j}$. By continuity it is clear that

$$
(-1)^{n-j} \frac{\partial^{j} G\left(\xi_{j}^{*}, t\right)}{\partial x^{j}} \leq 0
$$

and that $\xi_{j}^{*}<t-\delta$. From (2.34), (2.35) and the mean value theorem there must be a $\left.\xi_{j+1} \in\right] \xi_{j}^{*}, t-\delta[$ such that

$$
(-1)^{n-j-1} \frac{\partial^{j+1} G\left(\xi_{j+1}, t\right)}{\partial x^{j+1}}<0 .
$$


We can apply this argument recursively until finding $\left.\xi_{n} \in\right] \xi_{n-1}^{*}, t-\delta[$ such that

$$
\frac{\partial^{n} G\left(\xi_{n}, t\right)}{\partial x^{n}}<0
$$

where $\xi_{n}>\xi_{i}^{*}$ for $j \leq i \leq n-1$. However, the definition of $\xi_{i}^{*}$ implies

$$
(-1)^{n-i} \frac{\partial^{i} G\left(\xi_{n}, t\right)}{\partial x^{i}}>0, \quad k \leq i \leq n-1,
$$

which combined with (1.7), (2.20), (2.24) and the hypotheses (2.21) and (2.23) gives

$$
\frac{\partial^{n} G\left(\xi_{n}, t\right)}{\partial x^{n}}=-a_{n-1}\left(\xi_{n}\right) \frac{\partial^{n-1} G\left(\xi_{n}, t\right)}{\partial x^{n-1}}-\cdots-a_{0}\left(\xi_{n}\right) G\left(\xi_{n}, t\right) \geq 0,
$$

contradicting (2.36). This completes the proof.

Theorem 4. Let us fix $t \in] a, b[$ and let us assume that

$$
\begin{array}{ll}
(-1)^{n-k} a_{i}(x) \geq 0, \quad x \in[a, b], & i=0, \ldots, k-1, \\
(-1)^{n-i} a_{i}(x) \geq 0, \quad x \in[a, b], & i=k, \ldots, n-1
\end{array}
$$

and there is $a j<k$ such that $(-1)^{n-k} a_{j}(x)>0$ on $[a, b]$. Then, the $k$-th partial derivative of the Green function of (2.20) with $x$ changes sign in $] a, t[$.

Proof. Following a similar argument to the first part of Theorem 3 one has

$$
(-1)^{n-k} \frac{\partial^{k} G(x, t)}{\partial x^{k}}<0, \quad x \in[t, b[.
$$

The continuity of $\frac{\partial^{k} G(x, t)}{\partial x^{k}}$ for $x \in[a, b],(2.1)$ and (2.37) yield the result.

Remark 1. Theorems 3 and 4 extend the results of Peterson [15] (also in [1, Theorem 1.11.3]) on the sign properties of the Green function of the problem

$$
\begin{aligned}
& \left.y^{(n)}+\lambda p(x) y=0, \quad x \in\right] a, b[ \\
& y^{(i)}(a)=0, \quad i=0,1,2, \ldots, k-1, \\
& y^{\left(\beta_{i}\right)}(b)=0, \quad\left(\beta_{1}, \beta_{2}, \ldots, \beta_{n-k}\right)=(k, k+1, \ldots, n-1),
\end{aligned}
$$

with $\lambda= \pm 1$ and $p(x)>0$ on $[a, b]$, to the broader problem (2.20).

Theorem 5. Let $I(x, t)$ be defined as

$$
I(x, t)=\frac{\partial G(x, t)}{\partial a}, \quad(x, t) \in[a, b] \times[a, b] .
$$

Under the conditions (2.21)-(2.23) one has

$$
\begin{aligned}
& \left.\left.\left.(-1)^{n-k} \frac{\partial^{i} I(x, t)}{\partial x^{i}}<0, \quad(x, t) \in\right] a, b\right] \times\right] a, b[, \quad i=0, \ldots, k-1 \\
& (-1)^{n-i} \frac{\partial^{i} I(x, t)}{\partial x^{i}}<0, \quad(x, t) \in[a, b[\times] a, b[, \quad i=k, \ldots, n-1 .
\end{aligned}
$$


Proof. Let us fix $t \in] a, b[$. With the argument of [5, Lemma 3.3] $I(x, t)$ satisfies the BVP

$$
\begin{aligned}
& L I=0, \quad x \in[a, b] \\
& \frac{\partial^{i} I(a, t)}{\partial x^{i}}=0, \quad i=0,1,2, \ldots, k-2 ; \quad \frac{\partial^{k-1} I(a, t)}{\partial x^{k-1}}=-\frac{\partial^{k} G(a, t)}{\partial x^{k}}, \\
& \frac{\partial^{i} I(b, t)}{\partial x^{i}}=0, \quad i=k, \ldots, n-1 .
\end{aligned}
$$

Applying [8, Lemma 2.3 (i)-(ii)] to the problem (2.40)-(2.42) and using (2.1) one gets (2.38). The proof of (2.39) can be obtained following the same argument of the first part of Theorem 3 and taking (2.21)-(2.23) and (2.38) into account.

Theorem 6. Let $H$ be defined as in (2.2). Then under the conditions (2.21)(2.23) one has

$$
\begin{aligned}
& \left.\left.\left.(-1)^{n-k} \frac{\partial^{i} H(x, t)}{\partial x^{i}}>0, \quad(x, t) \in\right] a, b\right] \times\right] a, b[, \quad i=0, \ldots, k-1 \\
& (-1)^{n-i} \frac{\partial^{i} H(x, t)}{\partial x^{i}}>0, \quad(x, t) \in[a, b[\times] a, b[, \quad i=k, \ldots, n-1 .
\end{aligned}
$$

Proof. Let us fix $t \in] a, b[$. Again, with the argument of [5, Lemma 3.3] $H(x, t)$ satisfies the BVP

$$
\begin{aligned}
& L H=0, \quad x \in[a, b] \\
& \frac{\partial^{i} H(a, t)}{\partial x^{i}}=0, \quad i=0,1,2, \ldots, k-1 \\
& \frac{\partial^{i} H(b, t)}{\partial x^{i}}=0, \quad i=k, \ldots, n-2 ; \quad \frac{\partial^{n-1} H(b, t)}{\partial x^{n-1}}=-\frac{\partial^{n} G(b, t)}{\partial x^{n}} .
\end{aligned}
$$

From the proof of Theorem 3 and under the conditions $(2.21)-(2.23)$

$$
\frac{\partial^{n} G(b, t)}{\partial x^{n}}>0
$$

Applying recursively [8, Lemma 2.3] one obtains (2.43). And from (1.7), (2.20), $(2.21)-(2.23),(2.43)$ and the argument of the first part of the proof of Theorem 3 one finally gets to $(2.44)$.

\section{A procedure to assess solvability of the BVP}

Let us consider the eigenvalue problem

$$
M u(x)=\lambda u(x), \quad x \in[a, b]
$$

with $M$ defined as in (1.8). For the sake of completeness we will recall the main result of [2] on the problem (3.1): 
Theorem 7. [2, Theorem 2]. Let us suppose that there is a Banach space $B$ and a reproducing cone $P$ therein for which $M(P) \subset P$ and $M$ is $u_{0}$ positive. Then the eigenvalue problem (3.1) has a solution $u \in P$ and its associated eigenvalue $\lambda$ is positive, simple and bigger in absolute value than any other eigenvalue of such a problem. In addition, if the spectral radius of $M, r(M)$, is strictly increasing with the length of the interval $[a, b]$ (that is, if fixed $a, r\left(M_{a b}\right)$ is increasing with $b$ and fixed $b, r\left(M_{a b}\right)$ is decreasing with $a$ ) and $\lim _{b \rightarrow a^{+}} r\left(M_{a b}\right)=\lim _{a \rightarrow b^{-}} r\left(M_{a b}\right)=0$, one has:

- If there is no non-trivial solution of (1.4)-(1.6) at extremes $a^{\prime}, b^{\prime}$ equal or interior to $a, b$, then

$$
\lim _{k \rightarrow \infty} M^{k} v=0
$$

for any $v \in P$, for any $v, w \in P \backslash\{0\}$ there exists a $k_{0} \geq 1$ such that

$$
M^{k} v \leq M w, \quad k \geq k_{0}
$$

and there cannot be any $v \in P \backslash\{0\}$ and any $k_{1} \geq 1$ such that

$$
M^{k_{1}} v \geq v \text {. }
$$

- If there is a non-trivial solution of (1.4)-(1.6) at extremes $a^{\prime}, b^{\prime}$ interior to $a, b$, then for any $v, w \in P \backslash\{0\}$ there exists a $k_{2} \geq 1$ such that

$$
M^{k} v \geq M w, \quad k \geq k_{2}
$$

and there cannot be any $v \in P \backslash\{0\}$ and any $k_{3} \geq 1$ such that

$$
M^{k_{3}} v \leq v \text {. }
$$

The results of the previous section allow distinguishing two cases in the analysis of the problem (1.4)-(1.6) with regards to the application of Theorem 7 , depending on the number of derivatives of $G(x, t)$ whose sign is known, namely: 1 . The case $\beta_{1}<k, 2$. The case $\beta_{1}=k$.

Accordingly we will tackle both cases separately, focusing this section on the case $\beta_{1}<k$. Thus, let us assume first that $\mu \leq \beta_{1}<k$, let us pick $\nu$ such that $\mu \leq \nu \leq \beta_{1}$ and let us define the Banach space $B$ as

$$
B=\{y \in P C[a, b]\},
$$

if $\nu=0$, and as

$$
B=\left\{y \in C^{\nu-1}[a, b]: y^{(\nu)}(x) \in P C[a, b], y^{(i)}(a)=0, \quad 0 \leq i \leq \nu-1\right\},
$$

if $\nu>0$, in both cases the associated norm being

$$
\|y\|=\max \left\{\sup \left\{\left|y^{(i)}(x)\right|, \quad x \in[a, b]\right\}, i=0, \ldots, \nu\right\} .
$$

Let us also define the cone $P$ by

$$
P=\left\{y \in B:(-1)^{n-k} y^{(\nu)}(x) \geq 0 ; x \in[a, b]\right\} .
$$

From the definition of $P$ it is clear that $(-1)^{n-k} y^{(i)}(x) \geq 0, x \in[a, b], 0 \leq i \leq \nu$. With the help of the cone $P$ it is possible to prove the following theorem: 
Theorem 8. Let us assume that $\mu \leq \beta_{1}<k$ and

$$
(-1)^{n(\beta, j)} a_{j}(x) \leq 0, \quad x \in[a, b], j=0, \ldots, n-1, \quad j \neq \beta_{i}, i=1, \ldots, n-k
$$

with at least one $j$ such that $0 \leq j \leq n-1, j \neq \beta_{i}, i=1, \ldots, n-k$ and

$$
(-1)^{n(\beta, j)} a_{j}(x)<0, \quad \text { a.e. for } x \in[a, b] .
$$

Let us also assume that

$$
(-1)^{n-k} c_{j}(x) \geq 0, \quad x \in[a, b], j=0, \ldots, \mu
$$

with at least one $l \leq \mu$ such that

$$
(-1)^{n-k} c_{l}(x)>0, \text { a.e. for } x \in[a, b] .
$$

Then the conclusions of Theorem 7 are applicable to the problem (1.4)-(1.6) and the cone $P$ defined in (3.3).

Proof. The proofs of the reproducing character of $P$ and the $u_{0}$-monotonicity of $M$ on $P$ follow the same steps of [3, Theorem 2], replacing $\mu$ by $\nu$. The proof of the monotonicity of the eigenvalue $\lambda$ of (3.1) with $a$ mimicks that of $[2$, Theorem 8$]$, and the one with $b$ can be proven following the steps of $[7$, Theorem 8] and noting that, from Theorem 1, Theorem 2, (3.4) and (3.5), for $B<b$ one has

$$
\left.(-1)^{n-k} \frac{\partial^{i} G_{a b}(x, t)}{\partial x^{i}}>(-1)^{n-k} \frac{\partial^{i} G_{a B}(x, t)}{\partial x^{i}}>0,0 \leq i \leq \nu,(x, t) \in\right] a, b[\times] a, b[.
$$

Remark 2. Theorem 8 implies in practice that one can find bounds for the values of $a^{\prime}$ and $b^{\prime}$ for which (1.4)-(1.6) has a non-trivial solution, by assessing whether $M^{j} w \leq M^{i} w$ or $M^{j} w \geq M^{i} w$ for $j>i \geq 0$ and $w \in P \backslash\{0\}$. These bounds converge to $a^{\prime}$ and $b^{\prime}$ as the recursivity index $j$ grows.

As shown in [3], in the case that $\mu<\beta_{1}$ one can pick $\nu<\beta_{1}$ and functions $w \in P$ that simplify the practical evaluation of whether $M^{j} w \leq w$ or $M^{j} w \geq w$.

Theorem 9. Under the conditions of Theorem 8, suppose that $\mu \leq \nu<\beta_{1}$ and that $w \in P \backslash\{0\}$ is such that $w^{(\nu+1)}(x)$ exists and satisfies $(-1)^{n-k} w^{(\nu+1)}(x) \leq$ 0 on the points of $[a, b]$ where $w^{(\nu)}(x)$ is continuous. One has:

1. If $w(x) \in C^{\nu}[a, b]$ and there exists an integer $j>0$ such that

$$
(-1)^{n-k} w^{(\nu)}(b) \geq(-1)^{n-k} \frac{\partial^{\nu} M^{j} w(b)}{\partial x^{\nu}},
$$

then the problem (1.4)-(1.7) cannot have a non-trivial solution at extremes $a^{\prime}, b^{\prime}$ interior to $a, b$. 
2. If $(-1)^{n-k} w^{(\nu)}(a) \leq 0$, there exists a single discontinuity point $z \in[a, b]$ of $w^{(\nu)}(x)$ such that

$$
\lim _{x \rightarrow z^{-}}(-1)^{n-k} w^{(\nu)}(x)<(-1)^{n-k} w^{(\nu)}(z)=\lim _{x \rightarrow z^{+}}(-1)^{n-k} w^{(\nu)}(x)
$$

and there exists an integer $j>0$ such that

$$
(-1)^{n-k} w^{(\nu)}(z) \leq(-1)^{n-k} \frac{\partial^{\nu} M^{j} w(z)}{\partial x^{\nu}},
$$

then the problem (1.4)-(1.7) does have a non-trivial solution either at a, $b$ or at extremes $a^{\prime}, b^{\prime}$ interior to $a, b$.

Proof. The proof is identical as that of [3, Theorem 3] replacing $\mu$ by $\nu$ and noting that $(-1)^{n-k} \frac{\partial^{\nu+1} G(x, t)}{\partial x^{\nu+1}} \geq 0$ from Theorem 1 and that $\nu+1 \leq \beta_{1}$.

An example of a function $w(x)$ satisfying the conditions of the statement 1 of the Theorem 9 is

$$
w(x)=(-1)^{n-k} \frac{(x-a)^{\nu}}{\nu !}, \quad x \in[a, b] .
$$

And, picking any $z \in] a, b[$, an example of a function $w(x)$ that satisfies the conditions of the statement 2 of the Theorem 9 is

$$
w(x)= \begin{cases}0, & a \leq x<z, \\ (-1)^{n-k} \frac{(x-z)^{\nu}}{\nu !}, & z \leq x \leq b .\end{cases}
$$

Remark 3. As pointed out in the introduction, (1.4)-(1.7) is just a way of representing a set of problems of the type (1.1)-(1.3) in a way that allows applying the procedures of Theorems 7 or 9 . But as long as $p_{i}(x)=a_{i}(x)-c_{i}(x)$, $0 \leq i \leq \mu$, there are infinitely many choices of $a_{i}(x)$ and $c_{i}(x)$ that lead to problems for which the application of these Theorems, as the number of times $M$ is applied grows, yields bounds $a$ and $b$ that converge to the values $a^{\prime}$ and $b^{\prime}$ for which (1.1)-(1.3) has a non-trivial solution. Thus, one can wonder what choices of such functions facilitate a faster convergence to $a^{\prime}$ and $b^{\prime}$. [2, Theorem 12 and Remark 13] and [3, Remark 5] answered that question for the problem similar to (1.1)-(1.3) with $\beta_{n-k}<n-1$ by proving the faster convergence of the method when the functions $c_{i}(x), 0 \leq i \leq \mu$ are closer to zero. It is easy to prove that such a behaviour is also applicable to the cone $P$ defined in (3.3) and the problem (1.1)-(1.3), following the same steps used in $[2$, Theorem $12]$.

\section{The solvability of the purely right focal case}

When $\beta_{1}=k$ one can, under certain circumstances, extend the applicability of Theorem 7 to the case $\mu>\beta_{1}$, overcoming one of the main limitations of the method, as discussed in the conclusions of [2]. That is possible due to the constant sign of the partial derivatives of the Green function $G(x, t)$ with 
regards to $x$ under the conditions of Theorem 3. To this end, let us pick $\nu$ such that $k \leq \mu \leq \nu \leq n-1$ and let us define the Banach space $B$ as

$$
B=\left\{y \in C^{k-1}[a, b]: y^{(k)}(x) \in P C[a, b], y^{(i)}(a)=0, \quad 0 \leq i \leq k-1\right\},
$$

if $\nu=k$, and

$$
\begin{gathered}
B=\left\{y \in C^{\nu-1}[a, b]: y^{(\nu)}(x) \in P C[a, b], y^{(i)}(a)=0, \quad 0 \leq i \leq k-1,\right. \\
\left.y^{(i)}(b)=0, \quad k \leq i \leq \nu-1\right\},
\end{gathered}
$$

if $\nu>k$, in both cases with the associated norm

$$
\|y\|=\max \left\{\sup \left\{\left|y^{(i)}(x)\right|, \quad x \in[a, b]\right\}, i=0, \ldots, \nu\right\},
$$

as well as the cone $P$

$$
P=\left\{y \in B:(-1)^{n-\nu} y^{(\nu)}(x) \geq 0 ; x \in[a, b]\right\} .
$$

From the definition of $P$ one has $(-1)^{n-i} y^{(i)}(x) \geq 0, k \leq i \leq \nu$, as well as $(-1)^{n-k} y^{(i)}(x) \geq 0,0 \leq i \leq k-1$, for $x \in[a, b]$.

Theorem 10. Let us suppose that $\beta_{1}=k$ and

$$
\begin{aligned}
& (-1)^{n-k} a_{j}(x) \leq 0, \quad x \in[a, b], \quad j=0, \ldots, k-1, \\
& (-1)^{n-j} a_{j}(x) \leq 0, \quad x \in[a, b], \quad j=k, \ldots, n-1, \\
& (-1)^{n-k} a_{i}(x)<0, \quad \text { a.e. on }[a, b],
\end{aligned}
$$

for at least one $i$ such that $0 \leq i \leq k-1$. Let us also suppose that

$$
\begin{aligned}
& (-1)^{n-k} c_{j}(x) \geq 0, \quad x \in[a, b], \quad j=0, \ldots, k-1, \\
& (-1)^{n-j} c_{j}(x) \geq 0, \quad x \in[a, b], j=k, \ldots, n-1
\end{aligned}
$$

with at least one $l \leq n-1$ such that either

$$
(-1)^{n-k} c_{l}(x)>0, \text { a.e. on }[a, b],
$$

if $l<k$, or

$$
(-1)^{n-l} c_{l}(x)>0, \text { a.e. on }[a, b],
$$

if $l \geq k$. Then the conclusions of Theorem 7 are applicable to the problem (1.4)-(1.6) and the cone $P$ defined in (4.1).

Proof. Using the notation

$$
\begin{aligned}
& \{u(x)\}^{+}=(-1)^{n-\nu} \max \left\{(-1)^{n-\nu} u(x), 0\right\}, \\
& \{u(x)\}^{-}=(-1)^{n-\nu} \max \left\{-(-1)^{n-\nu} u(x), 0\right\}
\end{aligned}
$$

for $x \in[a, b]$, it is clear that

$$
y^{(\nu)}(x)=\left\{y^{(\nu)}(x)\right\}^{+}-\left\{y^{(\nu)}(x)\right\}^{-}, \quad x \in[a, b] .
$$


Then it is straightforward to show that, for any $y \in B$,

$$
\begin{aligned}
y(x) & =-\int_{a}^{x} \frac{(x-t)^{k-1}}{(k-1) !} \int_{t}^{b} \frac{(t-s)^{\nu-k-1}}{(\nu-k-1) !}\left\{y^{(\nu)}(s)\right\}^{+} d s d t \\
& +\int_{a}^{x} \frac{(x-t)^{k-1}}{(k-1) !} \int_{t}^{b} \frac{(t-s)^{\nu-k-1}}{(\nu-k-1) !}\left\{y^{(\nu)}(s)\right\}^{-} d s d t, \quad x \in[a, b]
\end{aligned}
$$

with the two terms of the right hand side being functions which belong to $P$. This proves that $P$ is a reproducing cone in $B$. The proof of the $u_{0}$-monotonicity of $M$ on $P$ is similar to that [3, Theorem 2], replacing $\mu$ by $\nu$ and applying Theorem 3, (2.29) and (4.2)-(4.8) to show that $M P \subset \mathcal{P}^{0}$, where $\mathcal{P}$ is a cone on the Banach space $\mathcal{B}$

$$
\begin{gathered}
\mathcal{B}=\left\{y \in C^{n}[a, b], y^{(i)}(a)=0, \quad 0 \leq i \leq k-1,\right. \\
\left.y^{(i)}(b)=0, \quad k \leq i \leq n-1\right\}
\end{gathered}
$$

such that

$$
\mathcal{P}=\left\{y \in \mathcal{B}:(-1)^{n-\nu} y^{(\nu)}(x) \geq 0 ; x \in[a, b]\right\},
$$

whose interior $\mathcal{P}^{0}$ is defined by

$$
\mathcal{P}^{0}=\left\{y \in \mathcal{B}:(-1)^{n-\nu} y^{(\nu)}(x)>0 ; x \in\left[a, b\left[, \quad y^{(n)}(b)>0\right\} .\right.\right.
$$

Obviously $\mathcal{P} \subset P$ since $\mathcal{B} \subset B$. Next, the monotonicity of the eigenvalue $\lambda$ of (3.1) with the extreme $a$ can be proven mimicking the one of [2, Theorem 8] and taking into account Theorem 5 and the hypotheses (4.2)-(4.8). And finally the monotonicity of $\lambda$ with $b$ can also be proven following the steps of $[7$, Theorem 8] and using Theorem 6 and the hypotheses (4.2)-(4.8).

Remark 4. The results of [3, Section 3] and in particular [3, Theorem 8] are also applicable to the problem (1.4)-(1.7) (whether $\beta_{1}<k$ or $\beta_{1}=k$ ) and provide a mechanism to apply Theorems 8 and 10 (that is, to determine whether $M^{j} w \geq$ $M^{i} w$ or $\left.M^{j} w \leq M^{i} w\right)$ when the comparison of $M^{j} w$ and $M^{i} w$ with respect to the cone is performed only at the points of a partition $\left\{x_{i}\right\}$ of $[a, b]$. This happens, for instance, when the calculation of $M^{j} w, M^{i} w$ and their derivatives is done via numerical integration.

As happened in the previous section, for the case that $k \leq \mu<n-1$ one can pick $\nu$ with $\mu \leq \nu<n-1$ and obtain a theorem equivalent to Theorem 9 .

Theorem 11. Under the conditions of Theorem 10, let us suppose that $\mu, k \leq$ $\nu<n-1$ and that $w \in P \backslash\{0\}$ is such that $w^{(\nu+1)}(x)$ exists and satisfies $(-1)^{n-\nu-1} w^{(\nu+1)}(x) \leq 0$ on those points of $[a, b]$ where $w^{(\nu+1)}(x)$ is continuous. One has:

1. If $w(x) \in C^{\nu}[a, b]$ and there exists an integer $j>0$ such that

$$
(-1)^{n-\nu} w^{(\nu)}(a) \geq(-1)^{n-\nu} \frac{\partial^{\nu} M^{j} w(a)}{\partial x^{\nu}},
$$

then the problem (1.4)-(1.7) cannot have a non-trivial solution at extremes $a^{\prime}, b^{\prime}$ interior to $a, b$. 
2. If $(-1)^{n-\nu} w^{(\nu)}(b) \leq 0$, there exists a single discontinuity point $z \in[a, b]$ of $w^{(\nu)}(x)$ such that

$$
\lim _{x \rightarrow z^{-}}(-1)^{n-\nu} w^{(\nu)}(x)=(-1)^{n-\nu} w^{(\nu)}(z)>\lim _{x \rightarrow z^{+}}(-1)^{n-\nu} w^{(\nu)}(x)
$$

and there exists an integer $j>0$ such that

$$
(-1)^{n-\nu} w^{(\nu)}(z) \leq(-1)^{n-\nu} \frac{\partial^{\nu} M^{j} w(z)}{\partial x^{\nu}}
$$

then the problem (1.4)-(1.7) does have a non-trivial solution either at a, $b$ or at extremes $a^{\prime}, b^{\prime}$ interior to $a, b$.

Proof. The proof is similar to that of [3, Theorem 3] replacing $\mu$ by $\nu$ and noting that $\frac{\partial^{\nu} M^{j} w(b)}{\partial x^{\nu}}=0$ (this follows from the boundary conditions at $b$ ) and that $(-1)^{n-\nu-1} \frac{\partial^{\nu+1} G(x, t)}{\partial x^{\nu+1}} \geq 0$, from Theorem 3 and the fact that $\nu+1 \leq n-1$, so that $(-1)^{n-\nu} \frac{\partial^{\nu+1} M^{j} w(x)}{\partial x^{\nu+1}} \leq 0$ for $x \in[a, b]$.

The conditions of the statement 1 of Theorem 11 are fulfilled, e.g., by

$$
w(x)=(-1)^{n-\nu} \int_{a}^{x} \frac{(x-t)^{k-1}}{(k-1) !} \frac{(t-b)^{\nu-k}}{(\nu-k) !} d t, \quad x \in[a, b] .
$$

And, picking a $z \in] a, b[$, the conditions of the statement 2 of Theorem 11 are fulfilled by

$$
w(x)= \begin{cases}(-1)^{n-\nu} \int_{a}^{x} \frac{(x-t)^{k-1}}{(k-1) !} \frac{(t-z)^{\nu-k}}{(\nu-k) !} d t & x \in[a, z] \\ (-1)^{n-\nu} \int_{a}^{z} \frac{(x-t)^{k-1}}{(k-1) !} \frac{(t-z)^{\nu-k}}{(\nu-k) !} d t & x \in] z, b]\end{cases}
$$

\section{Some examples}

In this section we will present a couple of examples where the results of the Sections 3 and 4 will be used to provide progressively better upper and lower bounds of the extremes that make (1.4)-(1.7) have a non-trivial solution. One of them will cover the case $\beta_{1}<k$ and the other the case $\beta_{1}=k$. In all of them the extreme $a$ will be fixed, focusing the analysis on the extreme $b$. Up to 3 decimal figures will be provided for each bound of $b$. The integral calculations will be done numerically using a mesh $\left\{x_{l}\right\}$ and applying the trapezoidal rule in each of the subintervals $\left[x_{l}, x_{l+1}\right]$ of the mesh. This also includes the calculation of the derivatives $\left(M^{k} v\right)^{(m)}(x), 0 \leq m \leq \nu$, as these can be written as

$$
\left(M^{k} v\right)^{(m)}(x)=\int_{a}^{b} \frac{\partial^{m} G(x, t)}{\partial x^{m}} \sum_{j=0}^{\mu} c_{j}(t)\left(M^{k-1} v(t)\right)^{(j)} d t .
$$

Example 1. Let us consider the following boundary value problem

$$
y^{\prime \prime \prime}+4 y^{\prime}-x y=0, \quad x \in[0, b], \quad y(0)=y(b)=y^{\prime \prime}(b)=0 .
$$


A possible representation of (5.1) in a manner that yields an equation $L y=0$ right disfocal in the interval of interest is

$$
y^{\prime \prime \prime}+4 y^{\prime}=(-1)^{n-k} x y, \quad x \in[0, b], \quad y(0)=y(b)=y^{\prime \prime}(b)=0
$$

with $n=3, k=1, \beta_{1}=0, \mu=0$. This forces $\nu=0$ and prevents the use of Theorem 9 since $\nu=\beta_{1}$. Nevertheless, the problem satisfies the hypotheses of Theorem 8, which allows applying Theorem 7 to estimate the value of the extreme $b$. To do so, we need to calculate the Green function of the BVP

$$
y^{\prime \prime \prime}+4 y^{\prime}=0, \quad x \in[0, b], \quad y(0)=y(b)=y^{\prime \prime}(b)=0,
$$

which can be obtained following Coppel [4] as

$G(x, t)= \begin{cases}(-\cot 2 b+\cot 2 b \cos 2 t+\sin 2 t) \frac{\sin 2 x}{4}+\frac{\cos 2 x-1}{4}, & 0 \leq x \leq t \leq b, \\ \frac{\cos 2 t-1}{4} \cot 2 b \sin 2 x+\frac{1-\cos 2 t}{4} \cos 2 x, & 0 \leq t<x \leq b .\end{cases}$

In this example we have decided to test Theorem 7 with several functions $w(x)$ : the one defined in (3.6) and two others defined by

$$
w(x)= \begin{cases}0, & x \in\left[0, z_{1}[\cup] z_{2}, b\right] \\ 1, & x \in\left[z_{1}, z_{2}\right]\end{cases}
$$

one of them having $z_{1}=b / 3$ and $z_{2}=2 b / 3$ and the other one having $z_{1}=b / 4$ and $z_{2}=3 b / 4$. All these functions belong to the cone $P$ of (3.3).

The application of Theorem 7 (i.e., the comparison of $M^{j} w(x)$ and $M^{i} w(x)$ with respect to the cone $P$ for different values of $j, i)$ to these $w(x)$ yields the Table 1 . Since $M w(0)=M w(b)=0$ regardless of the selected $w(x)$, the comparison of $M w(x)$ and $w(x)$ can only provide lower bounds of $b$ when $w(x)$ is that of (3.6) and upper bounds of $b$ when $w(x)$ is that of (5.2). This limitation is not present for higher values of $j, i$.

Table 1. Comparison of bounds for $b$ in the Example 1.

\begin{tabular}{lllllll}
\hline & \multicolumn{2}{c}{ Bound with (3.6) } & $\begin{array}{l}\text { Bound with }(5.2) \\
\text { and } z_{2}-z_{1}=\frac{b}{2}\end{array}$ & $\begin{array}{l}\text { Bound with (5.2) } \\
\text { and } z_{2}-z_{1}=\frac{b}{3}\end{array}$ \\
\hline $\begin{array}{l}\text { Recursivity } \\
\text { index }\end{array}$ & $\begin{array}{l}\text { Lower } \\
\text { bound }\end{array}$ & $\begin{array}{l}\text { Upper } \\
\text { bound }\end{array}$ & $\begin{array}{l}\text { Lower } \\
\text { bound } \quad \begin{array}{l}\text { Upper } \\
\text { bound }\end{array}\end{array}$ & $\begin{array}{l}\text { Lower } \\
\text { bound }\end{array}$ & $\begin{array}{c}\text { Upper } \\
\text { bound }\end{array}$ \\
\hline $\mathrm{j}=1, \quad \mathrm{i}=0$ & $b>1.419$ & N.A. & N.A. & $b<1.51$ & N.A. & $b<1.52$ \\
$\mathrm{j}=2, \quad \mathrm{i}=1$ & $b>1.465$ & $b<1.469$ & $b>1.466$ & $b<1.469$ & $b>1.466$ & $b<1.469$ \\
$\mathrm{j}=3, \quad \mathrm{i}=2$ & $b>1.467$ & $b<1.468$ & $b>1.467$ & $b<1.468$ & $b>1.467$ & $b<1.468$ \\
\hline
\end{tabular}

Table 1 shows that, as expected, the bounds get improved when the number of iterations grows. The precise value of $b$ which makes (5.1) have a non-trivial solution lies between 1.467 and 1.468, and those bounds are obtained with just three iterations of $M$. All functions $w(x)$ give similar bounds, with slightly better results for the $w(x)$ of (5.2) and $j=2$. For $j=1$ the method provides a better upper bound for $b$ if we pick $z_{2}-z_{1}=\frac{b}{2}$ instead of $z_{2}-z_{1}=\frac{b}{3}$ in the function $w(x)$ of (5.2), but such an advantage is not clearly visible for other values of $j$, at least if we stick to three decimal figures in the calculation of $b$. 
Example 2. Let us consider the following boundary value problem associated to the Bessel equation of order zero:

$$
x^{2} y^{\prime \prime}+x y^{\prime}+x^{2} y=0, \quad x \in[a, b], \quad y(a)=y^{\prime}(b)=0,
$$

where $a$ is the first positive zero of the Bessel function of the first kind and order zero, i.e. $a=2.4048$. Since $J_{0}^{\prime}(x)=-J_{1}(x), b$ must be the first positive zero of the Bessel function of the first kind and order 1, i.e. $b=3.8317$. This will allow testing the speed of convergence of the method to the value of $b$.

We can rewrite the problem (5.3) as

$$
y^{\prime \prime}+y=-\frac{1}{x} y^{\prime}, \quad x \in[a, b], \quad y(a)=y^{\prime}(b)=0
$$

with $n=2, k=1, \beta_{1}=1$ and $\mu=1$, which prevents the use of Theorem 11 since one can only choose $\nu=1=n-1$. However, for $b<a+\frac{\pi}{2}$ the equation

$$
y^{\prime \prime}+y=0
$$

is disfocal on $[a, b]$, and the problem (5.4) satisfies all the hypotheses of Theorem 10, which allows the application of Theorem 7 to estimate the value of $b$. To do that we first need to determine the Green function of the problem

$$
y^{\prime \prime}+y=0, \quad x \in[a, b], \quad y(a)=y^{\prime}(b)=0 .
$$

Following Coppel [4] as we did in Example 1, the mentioned Green function can be calculated as

$$
G(x, t)= \begin{cases}-\sin (x-a) \frac{\cos (b-t)}{\cos (b-a)}, & a \leq x \leq t \leq b, \\ -\cos (b-x) \frac{\sin (t-a)}{\cos (b-a)}, & 0 \leq t<x \leq b\end{cases}
$$

The application of Theorem 7 (i.e., the comparison of $M^{j} w(x)$ and $M^{i} w(x)$ with respect to the cone $P$ for different values of $j, i)$ to the functions $w(x)$ defined in (4.9) and (4.10) (considering two different cases $z=a+\frac{b-a}{2}$ and $z=a+\frac{b-a}{3}$ to test the effect of the choice of $z$ in the result of the calculations) gives the Table 2. As happened in the previous example, the value of the selected $w^{(\nu)}(x)$ at the neighborhood of $b$ affects the bounds of $b$ that can be obtained by comparing $M w(x)$ and $w(x)$ : the $w(x)$ of (4.9) can only yield a lower bound of $b$, whereas the $w(x)$ of (4.10) can only yield upper bounds of $b$.

As expected, the bounds get improved when the number of iterations grows, the precise value of $b(b=3.8317$, let us recall) lying between 3.831 and 3.832 . The function $w(x)$ defined in (4.9) provides slightly better upper bounds than the $w(x)$ of $(4.10)$, regardless of the value of $z$, whereas with the lower bounds one has exactly the opposite behaviour, for all values of $j$. The use of $z=$ $a+\frac{b-a}{2}$ in (4.10) also provides better bounds (both lower and upper) than the use of $z=a+\frac{b-a}{3}$, for all values of $j$. It is also remarkable that with just three iterations one can obtain lower and upper bounds for the precise value of $b$ which are within a 0.001 distance of that value. 
Table 2. Comparison of bounds for $b$ in the Example 2.

\begin{tabular}{lllllll}
\hline & \multicolumn{2}{c}{ Bound with (4.9) } & \multicolumn{2}{c}{$\begin{array}{c}\text { Bound with }(4.10) \\
\text { and } z=a+\frac{b-a}{2}\end{array}$} & \multicolumn{2}{c}{$\begin{array}{l}\text { Bound with (4.10) } \\
\text { and } z=a+\frac{b-a}{3}\end{array}$} \\
\hline $\begin{array}{l}\text { Recursivity } \\
\text { index }\end{array}$ & $\begin{array}{l}\text { Lower } \\
\text { bound }\end{array}$ & $\begin{array}{l}\text { Upper } \\
\text { bound }\end{array}$ & $\begin{array}{l}\text { Lower } \\
\text { bound }\end{array}$ & $\begin{array}{l}\text { Upper } \\
\text { bound }\end{array}$ & $\begin{array}{l}\text { Lower } \\
\text { bound }\end{array}$ & $\begin{array}{c}\text { Upper } \\
\text { bound }\end{array}$ \\
\hline $\mathrm{j}=1, \quad \mathrm{i}=0$ & $b>3.663$ & N.A. & N.A. & $b<3.92$ & N.A. & $b<3.936$ \\
$\mathrm{j}=2, \quad \mathrm{i}=1$ & $b>3.814$ & $b<3.844$ & $b>3.82$ & $b<3.844$ & $b>3.818$ & $b<3.858$ \\
$\mathrm{j}=3, \quad \mathrm{i}=2$ & $b>3.83$ & $b<3.832$ & $b>3.831$ & $b<3.834$ & $b>3.83$ & $b<3.835$ \\
\hline
\end{tabular}

\section{Discussion}

The results presented in this paper allow extending the method described in [2] and [3], to the focal case $\beta_{n-k}=n-1$, and therefore can be used to calculate bounds for the extremes $a^{\prime}$ and $b^{\prime}$ for which the problem (1.1)-(1.3) has a nontrivial solution, bounds which converge to the concrete extremes $a^{\prime}$ and $b^{\prime}$ when the recursivity index of application of the operator $M$ grows. That extension comes at the expense of imposing sign conditions on the functions $a_{i}(x)$, conditions that were not needed in the non-focal problem. It is straightforward to show that the results can also be used to provide bounds for the principal eigenvalue of the problem (3.1) by comparing $M^{j} w$ and $\lambda^{j-i} M^{i} w$ with respect to the cone $P$.

In the application of the method there is freedom in the choice of the functions $a_{i}(x)$ and $c_{i}(x)$ that come from the decomposition $p_{i}(x)=a_{i}(x)-c_{i}(x)$, as long as some sign conditions on $a_{i}(x)$ and $c_{i}(x)$ are respected. As in [2], our recommendation is to look for $a_{i}(x)$ for which the corresponding problem (1.9) has a Green function easy to calculate and $c_{i}(x)$ as close to zero as possible. This guarantees a faster convergence of the method, as per Remark 3 .

The cases $\mu<\beta_{1}$ (for $\beta_{1}<k$ ) and $\mu<n-1$ (for $\beta_{1}=k$ ) allow the application of Theorems 9 and 11, which simplify the practical determination of $M^{j} w(x) \geq M^{i} w(x)$ with regards to the cone $P$ since they restrict the comparison to a single point instead of the whole interval $[a, b]$. In the rest of cases, if numerical integration is used over a mesh $\left\{x_{l}\right\}$ of $[a, b]$ it becomes necessary to include in the comparison an interpolation error for the interior of each of the subintervals $] x_{l}, x_{l+1}[$. Whereas the calculation of such an interpolation error can be sometimes tedious (it requires computing $\frac{\partial^{\nu+2} M^{j} w(x)}{\partial x^{\nu+2}}$, see Remark 4), its value is usually so small (it is proportional to the square of the step of the mesh) that it allows a faster convergence of the method using Theorem 7 even in the cases where Theorems 9 and 11 could be applied.

As for the choice of $w(x)$, the examples do not provide clear arguments for some or others, since the results depend on the type of searched bound. It is worth remarking, in any case, that in the assessed examples with just three iterations one could obtain bounds as precise as three decimal figures.

It is also worth remarking that the paper also provides results for the sign of the derivatives greater than $k-1$ of the Green function of the problem (2.20), in particular Theorem 3, which improve previous results as far as the 
authors are aware. They allow extending the method to problems where $\beta_{1}=k$ and $\mu>\beta_{1}$. The main limitation in this case is that one needs specific sign conditions for all $p_{i}(x)$, when the problem is displayed in the form (1.1)-(1.3), in order that all $a_{i}(x)$ and $c_{i}(x)$ satisfy the sign conditions required by the method. However, when these conditions are fulfilled, it allows picking $a_{i}(x)$ for which the calculation of the Green function is straightforward (e.g. $a_{i}(x)$ that are constant), which removes one of the problems of the method raised in the conclusions of [2].

\section{Acknowledgements}

This work has been supported by the Spanish Ministerio de Economia y Competitividad grant MTM2013-41765-P.

\section{References}

[1] R.P. Agarwal. Focal boundary value problems for differential and difference equations. Springer, Netherlands, 1998. https://doi.org/10.1007/978-94-017-1568-3. Mathematics and Its Applications, Vol. 436

[2] P. Almenar and L. Jódar. Solvability of $n$th order boundary value problems. International Journal of Differential Equations, 2015:1-19, 2015. https://doi.org/10.1155/2015/230405.

[3] P. Almenar and L. Jódar. Improving results on solvability of a class of $n$ th-order linear boundary value problems. International Journal of Differential Equations, 2016:1-10, 2016. https://doi.org/10.1155/2016/3750530.

[4] W.A. Coppel. Disconjugacy. Springer-Verlag, New York/Berlin, 1971. Lecture Notes in Mathematics, No. 220, first edition

[5] P.W. Eloe, D. Hankerson and J. Henderson. Positive solutions and conjugate points for multipoint boundary value problems. Journal of Differential Equations, 95(1):20-32, 1992. https://doi.org/10.1016/0022-0396(92)90041-K.

[6] P.W. Eloe and J. Henderson. Focal points and comparison theorems for a class of two point boundary value problems. Journal of Differential Equations, 103(2):375-386, 1993. https://doi.org/10.1006/jdeq.1993.1055.

[7] P.W. Eloe and J. Henderson. Focal point characterizations and comparisons for right focal differential operators. Journal of Mathematical Analysis and Applications, 181(1):22-34, 1994. https://doi.org/10.1006/jmaa.1994.1003.

[8] P.W. Eloe and J. Ridenhour. Sign properties of Green's functions for a family of two-point boundary value problems. Proceedings of the American Mathematical Society, 120(2):443-452, 1994. https://doi.org/10.2307/2159880.

[9] J.R. Graef and B. Yang. Positive solutions to a multi-point higher order boundary value problem. Journal of Mathematical Analysis and Applications, 316(2):409421, 2006. https://doi.org/10.1016/j.jmaa.2005.04.049.

[10] M. Greguš. Third Order Linear Differential Equations. Springer, Netherlands, 1987. https://doi.org/10.1007/978-94-009-3715-4. Mathematics and its Applications (East European Series), vol. 22.

[11] X. Hao, L. Liu and Y. Wu. Iterative solution to singular nth-order nonlocal boundary value problems. Boundary Value Problems, 2015(1):125, 2015. https://doi.org/10.1186/s13661-015-0393-6. 
[12] M.S. Keener and C.C. Travis. Positive cones and focal points for a class of nth order differential equations. Transactions of the American Mathematical Society, 237:331-351, 1978. https://doi.org/10.2307/1997625.

[13] M.A. Krasnosel'skii. Positive solutions of operator equations. Fizmatgiz, Moscow, 1962. English translation P. Noordhoff Ltd., Groningen, The Netherlands, 1964

[14] M.G. Krein and M.A. Rutman. Linear operators leaving invariant a cone in a Banach space. American Mathematical Society, New York, 1950.

[15] A.C. Peterson. Existence-uniqueness for focal-point boundary value problems. $\quad$ SIAM Journal of Mathematical Analysis, 12(2):173-185, 1981. https://doi.org/10.1137/0512018.

[16] K. Schmitt and H.L. Smith. Positive solutions and conjugate points for systems of differential equations. Nonlinear Analysis: Theory, Methods $\&$ Applications, 2(1):93-105, 1978. https://doi.org/10.1016/0362-546X(78)90045-7.

[17] Y. Sun, Q. Sun and X. Zhang. Existence and nonexistence of positive solutions for a higher-order three-point boundary value problem. Abstract and Applied Analysis, 2014:1-7, 2014. https://doi.org/10.1155/2014/513051.

[18] M. Feng X. Zhang and W. Ge. Existence and nonexistence of positive solutions for a class of nth-order three-point boundary value problems in Banach spaces. Nonlinear Analysis: Theory, Methods \& Applications, 70(2):584-597, 2009. https://doi.org/10.1016/j.na.2007.12.028. 